\title{
Hazards and Detection Techniques of Veterinary Drug Residues in Animal-Origin Food
}

\author{
Zhongyu Chen ${ }^{1}$ \\ ${ }^{1}$ Animal science, Murdoch University, Perth, WA 6150, Australia
}

\begin{abstract}
Veterinary drugs originally used to diagnose, prevent, control and treat animal diseases. However, now some of them can also be mixed into livestock feed as growth promoters. Although most countries have specified animal-derived veterinary drug residue standards, such as the maximum residue limit, withdrawal period and prohibition of some veterinary drugs as growth promoters, there are still some animal-derived products with illegal amount of drug residues. Consequently, long-term intake of products with excessive residues will result in irreversible impacts on human health, livestock industries and natural environment. To minimize these risks, the techniques of drug residues detection plays an important role in guarding food safety of animal products. Moreover, Veterinary drug residue detection technology, according to the author's opinion, can be divided into instrumental method, immunoassay method, biomolecular technology and bio-sensor technology. The purpose of this article is to collect and summarize the existing journal literature current about status, hazards and detection techniques of animal-origin veterinary drug residues so that ordinary readers can briefly understand the research status of the residue detection techniques, farmers can understand the importance of regulating and suitable handling of veterinary drugs, and relevant researchers may know about the emphasized difficulties that need to be overcome in drug residue detection techniques.
\end{abstract}

\section{Introduction}

Food safety has usually been the focus of the public. With the development of veterinary pharmacy, the emergency and improvement of veterinary drugs bring about the positive impact on the livestock and poultry industries probably, such as the control and prevention of disease. According to " Chinese Regulations on Administration of Veterinary Drugs", any edible animal products containing prohibited drugs or containing exceed standard drug residues are preventive to be sold. In addition, all permitted veterinary drugs have go through pharmacokinetics, safety, and efficacy tests before being released on the market. However, some farmers may apply high-dose veterinary drugs as growth promotors in pursuit of higher profits, which put customers at potential health hazard [1]. Therefore, it is significant to promote drug residue detection technology with high precision and quick testing speed. This article will review the status of the use of veterinary drugs, the harms of veterinary drug residues, and detection techniques.

Current Status of Veterinary Drug Usage, According to 《Veterinary Medicine and Pharmacy 》, the identification of veterinary drug is chemical compounds or composition for diagnosis, prevention, and control livestock diseases [2]. Therefore, the main purpose of Veterinary Drug Application is to maintain animals' health and or to be auxiliary in the treatment process such as anti-microbials, anti-parasite, anti-inflammatory [3]. Some veterinary drugs have also been added to livestock feed as growth promoters [4]. However, overuse and misuse of veterinary drugs may result in their residues in products of animal origin such as eggs, milk, meat products and honey [5][6]. For example, antibiotics are usually used to prevent infectious diseases during sensitive periods when animals may not be infected, also known as non-specific infection prevention [7]. This seems plausible, but since both infected and uninfected individuals are exposed to antibiotics and antibiotics are exposed to the environment more frequently as well. This handling is regarded as majority of antimicrobial resistance [3].

Globally, usage of veterinary pharmaceuticals is generally increasing, but its growth rate keeps dropping. The applying of veterinary drugs varies widely among countries due to different animals' species, livestock farming methods, and different climatic conditions. The commonly used types of medicine are antibiotics, antiparasitic drugs (endo-phytic and exo-phytic) and steroid hormones. Antibiotics account for $70 \%$ of the total animal drug consumption in the world [8]. Apart from the United States and New Zealand, it witnessed a descending tendency in usage antibiotics among most countries [8].Among them, tetracycline, for example, is still the most commonly used veterinary drug [9][8] because of its treatment of respiratory diseases (oxytetracycline, aureomycin, doxycycline, etc.). 
Therefore, they are still widely used in farm animals [10]. With gigantic consumption of veterinary antibiotics, there are rising concern in potential hazard on their management and detection. In detail, statistics in 2018 demonstrated that over half of all pharmaceuticals have been use, prescribed and dispense inappropriately in globe [6]. Additionally, it is estimated that total antimicrobial consumption will increase by $67 \%$ between 2010 and 2030 if no further limitation is taken. One article stated that One-third of the global growth in veterinary drug consumption is in developing countries[3].For reasons, firstly it probably because livestock husbandries structure in developing countries are transforming from the extensive farming to the intensive farming which contains low-dose but frequent applying of veterinary drugs [10]. Secondly, the emergence and use of generic drugs has increased the total consumption of antibacterial drugs and is related to the strength and spread of bacterial resistance [10].

The misusing of veterinary drugs can make them more difficult to treat animals and even human diseases. Some literature reviewers believes that although reducing the use of antibiotics in a population associate with reduction in drug resistance in the same population, there is currently little evidence linking the reduction in the use of antibiotics in livestock with the reduction of drugresistant micro-organisms in humans [9].However, abundance of literature shows that antibiotic resistance may transfer from animals to humans [8][11][12]. In order to protect public health, the EU has set maximum residue limits (MRLs) based on scientific assessment of the safety of drugs in animal foods, including all kinds of such chemicals in all production varieties[11].and addition strategy is withholding period, which refers to the time from the most recent use of veterinary drugs to the collection and consumption of eggs/milk and meat of such animals. For reasons, the suspension of antimicrobials is to prevent excessively high levels of harmful drugs from remaining in meat, milk and eggs. From the time of handling food animals to the time of slaughtering animals as food, the withdrawal period must be followed because there may be unmetabolized veterinary drug residue exceeds the allowable limit before the end of the withdrawal period. Such animal productions may be considered harmful to the human body or significantly increase bacterial resistance. Lastly recording of veterinary drugs is conducive to supervise global sale of veterinary drugs and related policy making. Although there are international-level organizations, such as the World Health Organization (WHO), the Food and Agriculture Organization (FAO) and the World Organization for Animal Health (OIE), to provide guidance documents and plans for building database of global veterinary drug distribution, there is no international treaty or agreement stipulates how to use or record them [9]. But the good news is that veterinary drug sales data in more countries than in the past 10 years are available in the public domain. These data can help locate the main types of veterinary drug residues in the natural environment in a country's specific environment [8]. However, the sales of veterinary drugs in several major antibiotic consumers such as China, India, and Canada are still unavailable [8].

\section{The hazards of veterinary drug residue}

Most antibiotics currently in use are relatively non-toxic even at higher concentration, and few of them can result in highly serious problems on public health. However, there would be irreversible effects on human health with long-term intake of animal-origin productions polluted by antibiotic residue[13][14].In detail, the effects can be divided into rising of antibiotics resistance in bacterial, toxicity, anaphylaxis, carcinogenesis, malformation and imbalance of intestinal bacterial colony. Additionally, leaking or emission of non-processed antibiotic into natural environment may lead to negative effects on ecological system[15][14].lastly, problem in food safety resulted from improper management and detection of veterinary drug residue may obstruct the development of livestock industries and global economy[2][16].

\section{1 risk at human health}

Some farms may use antibiotics as growth promotors. Although the usage is minor, long-period application still have risk of developing drug resistance [17][16]. One hand, such a strategy may directly decrease effects of veterinary drugs, which makes livestock disease more difficult to be treated. Moreover, farmers who lack the knowledge about veterinary pharmacy may use a larger number of drugs to rise the effects of treatment [16]. This vicious cycling may further strengthen antibiotics resistance. Then with wide usage of veterinary drug, antibiotics resistance gradually transformed from singledrug resistance to multi-drug resistance [18]. In "Analysis of the Impact of Veterinary Drug Residues on Animal Food Safety Based on Statistical Analysis", Yang li states that due to abuse of veterinary drugs, some commonly used antibiotics such as penicillin, chloramphenicol, gentamicin and part of sulfonamides, may completely lose their clinical curative function[19]. On the other hand, because of long time consuming program in innovation of antibiotics, if pathogenic bacterial are severely resistant to multiple antibiotics, the aquaculture industry will fall into a situation where no medicine is available, the number of illnesses may increase, and the number of deaths may increase significantly, finally affect the economic benefits on the farm.

Apart from rising the difficulty in livestock treatment, it was proved that antimicrobial resistance can transform from animals to humans. Bacteria can acquire resistance through direct contacting with the human body or indirect contacting with animal-origin products [2]. Indirect damage occurs through the production of multidrug resistant pathogens, which can be spread to humans by fertilizer contamination contain residual, or even through the soil contaminated by animal feces [21]. Damage to human health from drug-resistance strains is obvious. In Europe, multi-drug resistant bacteria such as methicillin-resistant staphylococcus aureus and bacteria 
contain $\beta$-lactamase were harmful to local public health [22]. According to statistics, the European Food Safety Authority reported that emergence of antibiotic resistance will cause about 25,000 deaths in EU member states every year and bring about 1.5 billion euros in healthcare costs and medical production losses [22]. Merve and Nurşen pointed out in 2019 that in the production process of animal-derived products, inappropriate and irresponsible using of antibiotics as growth promoters is a reason for the transfer of drug resistance to humans [21]. In 2005, the US Food and Drug Administration banned the use of certain antibiotics such as enrofloxacin in food animals due to the increased content of fluoroquinolone-resistant Campylobacter [23].

Excessive veterinary drug residues can cause poisoning when ingested into the human body [17]. Because of low residue of veterinary drug in animal products, acute poisoning is relatively rare, but long-term consumption of products containing veterinary drug residues will lead to chronic poisoning [15]. Various residual veterinary drugs may accumulate in human organs for a long time, and if they exceed a certain range, they will show obvious clinical symptoms. For example, chloramphenicol is a broad-spectrum antibacterial agent mainly used to fight infections caused by gram-negative bacteria [15]. Because it can cause aplastic anemia in human, it has been banned in the United States, the European Union and the United Kingdom. However, chloramphenicol hardly produces symptoms of poisoning in animals. The reason is that livestock have a strong adaptability to such antibiotics [24]. For another example, excessive sulfonamides residue can cause deposition of uric acid crystals in human kidneys and impair organ function [20]. Excessive streptomycin can cause damage to the human auditory system, and symptoms such as tinnitus and deafness often occur [20].

Antibiotics can also cause anaphylaxis in human. Antibiotics are usually hapten metabolites of antibiotics and some carrier tissues [24]. When the body's immune system attacks antibiotics, an allergic reaction to antibiotics will occur [24]. If the human body is allergic, such as having been exposed to antibiotics or after reingesting animal-derived products containing veterinary drugs, it may trigger an allergic reaction [15][18]. For example, antibiotic drugs such as sulfonamides, and some amino sugar antibiotics are prone to allergic reactions [20][18]. The most representative one is penicillin. Its allergic reaction can lead to asthma, digestive diseases, urticaria and even anaphylactic shock, which can be life-threatening [21]. In addition, allergic reactions to some antibiotics are more likely to occur in people who already have certain diseases. A study showed that the allergic reaction rate of sulfonamides is less than $1 \%$ in healthy people. However, in patients with hematological malignancies and AIDS patients, the effects of the drug's allergic reactions are as high as $12 \%-40 \%$ and $50 \%-60 \%$, respectively [25]. Finally, some allergic reactions can lead to further damage to organs. For example, a study showed that liver cell damage can be traced to the allergic reaction of macrolide antibiotics [2].
When veterinary drugs that induce mutations, malformations, and carcinogenesis enter the human body through animal food, the gradual accumulation of drugs will cause abnormalities in genes and chromosomes [17]. If too much residual veterinary drugs are taken, the accumulated gene mutation can contribute to cancer, malformations or mutations and other diseases [17]. For example, excessive estrogen, nitrofurans, and arsenic preparations in animal-derived products have carcinogenic effects. Benzimidazole drugs have teratogenic effects [18]. Secondly, some drugs, compounds or their metabolites can cause congenital malformations of the fetus during pregnancy [2]. For example, there are reports that aminoglycoside antibiotics can cause severe hearing damage or neurotoxicity to the developing fetus [26].

Finally, the impacts of veterinary drugs residues on the beneficial intestinal flora after entering the human body cannot be ignored. There are a variety of bacterial colonies in healthy human intestines, forming a relatively stable flora environment, which helps maintain human health [15][18]. Veterinary drug residues in animalderived products will disturbing normal colonies, thereby destroying the normal ecology in the intestinal tract [2]. When the symbiotic or normal colonies in the intestine are reduced, pathogenic microorganisms can grow and may cause infection [26]. At present, there are many reports demonstrating that broad-spectrum antibiotics are related to the infection of harmful bacteria, such as Bacillus carboxyformis [26][2].

\subsection{Hazard to natural environment}

Residues of veterinary drug can also enter the natural environment and cause indirect harm to the ecosystem or to the human body. In the process of livestock breeding, some veterinary drugs cannot be completely absorbed by animals, and may enter the natural environment through excrement, polluting water sources and soil [15][18][14]. Furthermore, veterinary drugs can infiltrate the ground with water, polluting the groundwater and resulting in a veterinary drug in drinking water sources, which then have a negative impact on human health [14]. Among them, benzo is the most harmful due to its difficulty in being digested [19]. Although some veterinary drugs, such as penicillin, are poorly stable under the action of microorganisms in soil and can be naturally decomposed [14], most of them can have a greater impact on the environment. For example, sulfadiazine in the soil can reduce the conversion of nitrogen in addition to reducing the activity of microorganisms. Oxytetracycline has the effect of inhibiting nitrification in simple aquatic systems [14].

\subsection{Blockage of development in aquaculture}

Veterinary drug residues can reduce the quality of livestock products and influence the effectiveness of market sales of livestock products. First of all, due to continuous increase of veterinary drug residues, overseas exports of livestock products in some areas have been 
severely blocked, which greatly affects the export of livestock products and the healthy and sustainable development of the livestock breeding industry [16].Beside, as a mounting people realize the potential risk of veterinary drug residues, such as cancer, fetal malformations, immune system abnormalities and other diseases, consumers may question the safety of animalsorigin production [27]. As a result, reducing consumption may cause dropping in farm production and may adversely impact global economy [27]. Meanwhile Last focused problem is that internationally, the maximum residue limit set by the Codex Alimentarius Commission has not been accepted by the national committee, resulting in different national standards for the maximum residue limit of veterinary drugs. This has led to the gradual decline in export of meat and animals' products [2].

\section{3 detection techniques of veterinary drug residue}

As mentioned above, veterinary drug residue generally has low concentration and can irreversibly damage human health by its accumulation [22]. Therefore, highefficiency detection technology plays an important role in food safety. Then author will introduce some commonly used detection methods from instrumental analysis, immunoassay, molecular biology technology and biosensor.

\subsection{Instrument Analysis Method}

Instrumental analysis method generally refer to the chromatographic or chromatographic mass spectrometry techniques to measure and detect target compound in samples [28]. because of well performance in reproducibility, sensitivity and selectivity, instrumental analysis methods have become a common method in drug residue detection [28]. the most representative instrumental analysis method is liquid chromatographymass spectrometry (LC-MS), Liquid chromatographytime-of-flight (LC-TOF) mass spectrometry and Liquid chromatography-ultraviolet (LC-UV) detection

The principle of LC-MS can be divided into two part: separation and analysis[29].In detail, the first part is separation of different compounds in sample which have distribution ratio in the liquid-solid or two immiscible liquids in chromatography system[30]. After sample is repeatedly adsorbed and decomposed in separation, concentration of the separated compound is transmitted into an electrical signal through the detector and, is recorded and displayed by Chromatogram [31]. At present, scientists have developed high-efficiency liquid chromatography (HPLC) and ultra-high-performance liquid chromatography (UHPLC) which combine liquid chromatography techniques with a high-pressure pump system [31]. These two detection methods increase speed of sampling, simplify the material separation process, and reduce the cost because chromatographic columns can also be reused [31][32]. The second part is the qualitative and quantitative analysis of the separated compounds by mass spectrometer [30]. The process is to ionize the target compounds and sort them according to the mass-to-charge ratio of each ion. This ratio is then is converted into a electrical signal by the detector [30][33]. After the signal is amplified, it is converted into a chromatogram of relationship between separation time and signal intensity by the data processing system, and quantitative analysis is carried out by calculating the chromatographic area [30][33]. LC-MS is a residue detection method with high accuracy, high sensitivity, and strong selectivity. It is suitable for the detection of multiple veterinary drug residues [29][28].

Studies have applied liquid LC-MS technology combined with acetonitrile extraction and strong cationic phase extraction column purification to detect 15 quinolone residues in water products [33]. The results showed that all quinolones had a good linear relationship in the range of $1.0-100 \mu \mathrm{g} / \mathrm{L}$, and the correlation coefficient was $0.9924-0.9992$. Within the concentration range of $0.002-0.040 \mathrm{mg} / \mathrm{kg}$, the average standard recovery rate is $79.9 \%-93.8 \%$ [33].

Hyo-Ju and Seung-Hak et al. used QuEChERS as a pretreatment and then used the LC-MS/MS method to detect 17 antibiotic residues in chicken meat in Korea [34]. Although the sample matrix is complex, the recoveries of 12 antibiotic samples are more than $90 \%$, most of which have relative standard deviations below $15 \%$, and the linear relationship (expressed by the square of the correlation coefficient) is greater than 0.98. No interference peaks were found in the chromatogram of the chicken. Compared with previous studies, the recoveries and relative standard deviations of most antibiotics in chicken meat have satisfactory results in this method. It also has advantages in sensitivity and detection limit (as low as $0.01 \mathrm{~g} / \mathrm{kg}$ ) [34].

Although LC-MS is widely used in the field of veterinary drug residue detection, some researchers believe that the relative molecular mass of the analyte given by liquid chromatography-mass spectrometry is low, and structural information is lacking, so multi-stage mass spectrometry technology is needed to improve [30]. Additionally, purity of separated chemicals can lead to inaccurate result [29].

Then, liquid chromatography-time-of-flight mass spectrometry (LC-TOF/MS) can also be applied to the detection of veterinary drug residues and has a good performance. The principle of LC-TOF/MS bases on that ions with different mass-to-charge ration have different time to pass through field-free region with constant speed [35]. In general, ions with smaller mass-to-charge ratio arrive the detector faster than the ions with larger massto-charge ratio. With this property, ions of different masses can be separated according to time reach detector [35].

In 2018, Belen and Laura et al. used optimized LCTOF/MS to simultaneously detect oxytetracycline, florfenicol and flumequine residues in seawater, marine sediments and marine creature samples [36]. The optimization part mainly contains use of liquid-liquid extraction and solid-liquid extraction, purification using silica and other commercial adsorbents, and single and tandem solid phase extraction procedures. The result 
illustrated 100-500 $\mathrm{mG} / \mathrm{KG}$ in invertebrates with good accuracy and precision. The recovery rate is between $65 \%$ and $120 \%$ [36].

Although LC-TOF/MS has high selectivity and sensitivity, its resolution is low due to ions dispersion in time and space before entering the drift tube [35].

Finally, high performance liquid chromatographyultraviolet (HPLC-UV) detection method is an important method for residue detection in food before innovation of LC-MS [37]. Through the separation and purification of the pretreatment technology, the UV detector, such as a diode array detector, is a method of detecting the residue by detecting the absorption of the solute to the ultraviolet light [37]. Because of its low detection cost and good selectivity and sensitivity, it has a relatively high number of users in China [37].

Juan and Federico et al. used QuEChERS as a pretreatment technique to optimize high-performance chromatography-UV detection to test three anti-coccidia veterinary drugs residued in chicken liver (lasalocid, dinitrofenidol and diclazuril) [38]. Experimental data shows that the average recovery rate of the target compound is $91 \%$, and the overall accuracy is $5.5 \%$. The recovery rates of lasalocid, dinitrazine, and diclazuril are $20 \mu \mathrm{g} / \mathrm{kg}, 15 \mu \mathrm{g} / \mathrm{kg}$ and $120 \mu \mathrm{g} / \mathrm{kg}$, respectively [38].

Donay and Ibrahim optimized the reverse HPLC-UV detection method, which can simultaneously detect the amount of sulfaclopyrazine and trimethoprim. This technology can be more accurate, fast, and cost-effective. The sample was separated by using a Hypersil GOLD ${ }^{\mathrm{TM}}$ C18 Selectivity HPLC column and acetonitrile with $\mathrm{pH}$ 3.0 buffer solution $(30: 70, \mathrm{v} / \mathrm{v})$ at a flow rate of 0.8 $\mathrm{ml} / \mathrm{min}$. And analyzed on a $272.0 \mathrm{~nm}$ ultraviolet detector. After optimization, the retention time of sulfaclopyrazine and trimethoprim were 4.37 and 2.60 minutes, respectively. This method has a good linear relationship in the range of $1.0 \sim 100.0$. Sulfachloropyrazine and trimethoprim are $0.5-40.0 \mu \mathrm{g} / \mathrm{ml}$ and $0.5-40.0 \mu \mathrm{g} / \mathrm{ml}$, respectively. The detection limits of sulfachloropyrazine and trimethoprim are $0.05 \mu \mathrm{g} / \mathrm{ml}$ and $0.18 \mathrm{~g} / \mathrm{ml}$, respectively [39].

Table 1 the Table about Recovery Rate and Limit of Detection of Instrument Analysis Method

\begin{tabular}{|c|c|c|c|c|}
\hline Reference & Detection Method & Detected Drug & Recovery rate & $\begin{array}{l}\text { LOD(limit of } \\
\text { detection) }\end{array}$ \\
\hline \multirow{3}{*}{$\begin{array}{l}\text { Hyo-Ju and Seung-Hak et } \\
\text { al. [34] }\end{array}$} & \multirow[t]{3}{*}{ LC-MS/MS } & amoxicillin & $82.4 \%-99.5 \%$ & $0.07 \mu \mathrm{g} / \mathrm{kg}$ \\
\hline & & ceftiofur & $89.4 \%-94.6 \%$ & $0.15 \mu \mathrm{g} / \mathrm{kg}$ \\
\hline & & tylosin & $84.3 \%-87.05 \%$ & $0.07 \mu \mathrm{g} / \mathrm{kg}$ \\
\hline \multirow[t]{3}{*}{ Belen and Laura et al. [36] } & \multirow{3}{*}{$\begin{array}{l}\text { optimized } \\
\text { TOF/MS }\end{array}$} & Oxytetracycline & $85 \%$ & $0.3 \mu \mathrm{g} / \mathrm{L}$ \\
\hline & & Florfenicol & $98 \%$ & $1.5 \mu \mathrm{g} / \mathrm{L}$ \\
\hline & & Flumequine & $108 \%$ & $0.3 \mu \mathrm{g} / \mathrm{L}$ \\
\hline \multirow[t]{3}{*}{$\begin{array}{l}\text { Juan and Federico et al. } \\
{[38]}\end{array}$} & \multirow[t]{3}{*}{$\begin{array}{l}\text { high-performance } \\
\text { chromatography-UV }\end{array}$} & $\begin{array}{l}\text { Lasalocid } \\
\text { (coccidiostats) }\end{array}$ & \multirow[t]{3}{*}{$>91 \%$} & $20 \mu \mathrm{g} / \mathrm{kg}$ \\
\hline & & $\begin{array}{l}\text { Nicarbazin } \\
\text { (coccidiostats) }\end{array}$ & & $15 \mu \mathrm{g} / \mathrm{kg}$ \\
\hline & & $\begin{array}{l}\text { Diclazuril } \\
\text { (coccidiostats) }\end{array}$ & & $120 \mu \mathrm{g} \mathrm{kg}-1$ \\
\hline \multirow[t]{2}{*}{ Donay and Ibrahim [39] } & \multirow[t]{2}{*}{ reverse HPLC-UV } & Sulfachloropyridazine & 1 & $0.05 \mathrm{~g} / \mathrm{ml}$ \\
\hline & & Trimethoprim & 1 & $0.18 \mathrm{~g} / \mathrm{ml}$ \\
\hline
\end{tabular}

\subsection{Immunoassay}

The immunoassay method is a relatively common way to inspect veterinary drugs in animal-origin products through immunological detection techniques [31].The theory of immunoassay is that the target antigen and the antigen that are labeled with enzymes or fluorescence compete for binding to antibody to present color reaction, and the color depth can reflect the content of the target compound in the sample[31]. Because most veterinary drugs are relatively small molecules and have weak immunogenicity, they react like hapten and need combine to large molecular weight carriers to form artificial antigens to achieve the purpose of detection [40][31][41]. Due to its high sensitivity, short detection time, and strong specificity, it is widely used in the detection of veterinary drug residues [31] [42]. Among the more commonly used technologies are enzymelinked immunosorbent technology (ELISA) and fluorescence immunoassay technology [40][31].
ELISA bases on specific immune response of antigen and antibody under the high efficient catalysis of enzymes to trigger color reaction of antigen-antibody [32][37].And targeted compounds can be qualified with depth of color. This method is fast and easy to operate, so it is suitable for mass screening of samples [32][37].

In 2019, Suzanne, Christelle and others used a competitive enzyme-linked immunosorbent technique (COMPETITIVE ELISA) to analyze the residues of tetracyclines and penicillin in different dairy products in Lebanese dairy brands [43]. The results showed that $90.9 \%$ of milk samples were positive for penicillin residues $(0.27-0.77 \mathrm{UG} / \mathrm{KG})$, but the detection results of tetracycline and penicillin residues in yogurt were below the detection limit of ELISA [43].

Akram and Alireza used indirect competitive ELISA in 2019 to detect the residues of ciprofloxacin and tetracycline in chicken and beef. The analysis found that ciprofloxacin residues could be detected in $100 \%$ beef and $95 \%$ chicken samples. The detection limits of ciprofloxacin and tetracycline in these two meat products 
were $0.525 \mathrm{ng} / \mathrm{g}$ and $1.5 \mathrm{ng} / \mathrm{g}$, respectively. However, the recovery rate was not mentioned in this study [44].

The current challenge of immunoassay in China is the lack of supervision of ELISA kits on the market. As poor quality or substandard ELISA kits appear on the market, their test results are inaccurate or even unable to detect veterinary drug residues [1].

In addition to enzyme-linked immunosorbent technology, fluorescence immunoassay technology is also one of the commonly used immunoassay methods [41]. It adopts the principle of energy level transition to produce fluorescence by giving electrons energy to complete the transition from the ground state to the excited state [32]. In this method, the antibody antigen is labeled with a fluorescent isotope, and the detection of the compound is achieved by checking its fluorescence intensity [41].

Table 2 the Table about the Recovery Rate and Limit of Detection of Immunoassay

\begin{tabular}{|l|l|l|l|l|}
\hline Reference & Detection Method & Detected Drugs & $\begin{array}{l}\text { Recovery } \\
\text { Rate }\end{array}$ & $\begin{array}{l}\text { LOD (limit of } \\
\text { detection) }\end{array}$ \\
\hline $\begin{array}{l}\text { Suzanne and } \\
\text { Christelle [43] }\end{array}$ & Competitive ELISA & Tetracycline & $/$ & $1.80 \mu \mathrm{g} / \mathrm{kg}$ \\
\cline { 3 - 5 } & & Penicillin & $/$ & $4.00 \mu \mathrm{g} / \mathrm{kg}$ \\
\hline $\begin{array}{l}\text { Akram and } \\
\text { Alireza [44] }\end{array}$ & Competitive ELISA & Tetracycline & $/$ & $1.5 \mathrm{ng} / \mathrm{g}$ \\
\cline { 2 - 5 } $\begin{array}{l}\text { Yuanze and Jie } \\
\text { et al [45] }\end{array}$ & TIME-RESOLVED & Streptomycin & $86.2 \sim 96.3 \%$ & $1.8 \mathrm{mg} / \mathrm{kg}$ \\
\hline
\end{tabular}

\subsection{Molecular Biological Technique}

In the detection of veterinary drug residues, the common molecular biology detection technologies are biochip and molecular imprinting technology. Firstly, the biochip technology uses the principle of specific interaction between molecules to rapidly process and detect biomolecules [32][46]. Specifically, biochips can sequentially fix biological macromolecules on silicon wafers, glass wafers, etc. After the arrangement is complete, it can react with the target compound and then be detected and analyzed by the scanner [46]. This method has an advantage on high throughput and rapid detection, which has gradually attracted the attention of researchers. In the detection of sulfamethazine residues in dairy products, the detection limit is low $(0.06 \mu \mathrm{G} / \mathrm{L})$, and the detection time is extremely short ( $<15 \mathrm{mins})$ [46].

Valerie and Celine et.al. used multiple immunoassays based on biochip technology to simultaneously screen multiple antibiotics in milk. The author names detection method as EVIDENCE INVETSIGATOR SYSTEM [47]. Its core is a miniature silica gel chip (biochip) containing discrete test areas for immobilized antibodies against different antibiotics [47]. An increase in the amount of antibiotics in the sample will result in a decrease in the binding of horseradish peroxidase-labeled antibiotics, resulting in a decrease in the chemiluminescence signal. Finally, the charge-coupled device camera associated with the imaging device performs luminescence detection. The test results show that this method has a fast detection speed and a low false positive rate [47].

Aksem also applied the same biochip multi-array technology (EVIDENCE INVETSIGATOR SYSTEM) to detect 12 types of antibiotic residues in honey in 2019.
Yuanze and Jie et al. used TIME-RESOLVED FLUOROIMMUNOASSAY to quantify the residual streptomycin in milk. This method uses a europium $(\mathrm{EU} 3+)$ chelate-labeled secondary antibody as a tracer, and trichloroacetic acid is used to deproteinize milk samples [45]. The results showed that the detection limit of streptomycin in milk was $1.8 \mathrm{mG} / \mathrm{KG}$, the sample recovery rate was $86.2-96.3 \%$, and the relative standard deviation was less than $11 \%$. The researchers compared the detection effect of this method and ultra-highperformance liquid chromatography-mass spectrometry (UHPL-LC/MS) on the same sample. The test results show that the data obtained by the two monitoring methods are similar, which proves that the time-resolved fluorescence immunoassay method can accurately detect streptomycin residues in milk [45].

The results showed that residues of erythromycin, streptomycin, neomycin, tylosin B, amikacin and lincomycin were found in honey samples at different concentrations. Among them, erythromycin can be detected in $91 \%$ of local honey samples [48].

At present, the biochip technology requires more research experiments to optimize in the field of biological residue detection. The main problem is that there are fewer types of drugs that can be tested, and it is difficult to achieve multi-residue testing [46]. In addition, VALÉRIE et al. also pointed out that each step of the multiple immunoassays of biochip technology requires artificial intervention and the high cost of detection equipment, which may make the method difficult to popularize [47].

In addition, molecular imprinting is more commonly used as a pretreatment technology and is widely used in the field of antibiotic residue detection [49]. The principle is to prepare polymers with a high degree of recognition for specific compound molecules through chemical methods [49][32]. The polymer used is polymerized with a polymer material from a template similar to the target molecule to form a material that can recognize a specific compound [49][32]. Molecularly imprinted polymers have strong specificity, stability and affinity, so that they can selectively adsorb specific compounds in samples with complex matrices [50]. The application of molecular imprinting technology in the detection of veterinary drug residues is mainly the packing in the solid-phase extraction column to achieve the enrichment, purification and separation of specific compounds. It is used as a stationary phase in chromatographic separation, as an identification element and enzyme in the sensor. Antibodies play the same role 
and are used in conjunction with immunological techniques to increase detection speed and sensitivity [49].

At present, the development direction of molecular imprinting technology is to increase the research and development of new methods to make its application more widely. In addition, the research of its combination with nanotechnology may realize the new high-speed detection technology of materials [49].

Table 3 the Table about the Recovery Rate and Limit of Detection of Molecular Biological Technique

\begin{tabular}{|l|l|l|l|l|}
\hline Reference & Detection method & Detected drug & $\begin{array}{l}\text { mean Recovery } \\
\text { rate }\end{array}$ & $\begin{array}{l}\text { (LOD, mean limit } \\
\text { of detection) }\end{array}$ \\
\hline \multirow{3}{*}{ Aksem[48] } & $\begin{array}{l}\text { biochip multi-array } \\
\text { technology }\end{array}$ & Spiramycin & $/$ & $2.0 \mathrm{ppb}$ \\
\cline { 3 - 5 } & & Apramycin & $/$ & $2.0 \mathrm{ppb}$ \\
\cline { 3 - 5 } & Bacitracin & $/$ & $1.2 \mathrm{ppb}$ \\
\cline { 3 - 5 } & Neomycin & $/$ & $1.0 \mathrm{ppb}$ \\
\hline
\end{tabular}

\subsection{Biosensor}

A biosensor is a biological identification element that uses biologically active substances [51]. When it is combined with a biological component, the physical or chemical signal it receives is converted into an electrical signal that can be transmitted and detected. At the same time, the electrical signal is amplified by a detection amplifier to achieve the detection of the target compound [51][52]. Its types are immunosensor, enzyme sensor and aptamer sensor [52]. With the continuous development of biotechnology, the accurate detection results and easy operation of biosensors have enabled them to have a wide range of applications in biological detection [37][51][30]. For example, Li Fang and Kang Huaibin reviewed in 2017 that electrochemical immunosensors have low detection limits and good detection ranges in detecting penicillin $\mathrm{G}$, chloramphenicol, fluoroquinolone in milk and sulfadiazine in honey [51]. $\mathrm{Li} \mathrm{Yu}$ summarized in the "Development and Application of Biosensors in Food Safety" that plasma resonance sensors have low detection lines in dairy products, shrimps, honey and pig kidneys and have good performance in the detection of veterinary drug residues in meat products. Performance [52]. Biosensors have developed rapidly in terms of detecting pesticide and veterinary drug residues. Currently main research direction is to increase the stability of antibody preparation in immunosensors, and to develop more antibiotic aptamers in aptamer sensors [51].

\section{Conclusion and Prospect}

In conclusion, the author makes the following suggestions on the future research. First, many documents reviewed by the author focused on the development, harm, and advantages of veterinary drug residue detection technology, and the corresponding types of veterinary drugs suitable for detection, but rarely mentioned the difficulty of popularization of detection technology. Many documents verify the high accuracy and detection speed of the new residue detection technology, but rarely mention the technology's penetration rate. If advanced technologies cannot be popularized smoothly, they may reduce the practicability of such technologies. For this reason, the author suggests conducting more in-depth research on how to reduce the difficulty and cost of veterinary drug residue detection technology. Second, in the hazards of veterinary drug residues, most of the literature mainly mentions its hazards to the human body and the environment, but seldom mentions its impact on the economy. It is recommended that the following research be biased towards its impact on the economy.

\section{References}

1. Qiuxia Z, Tao Z, Jinjin H. Detection Meothd and Classification of veterinary drug residue[J]. Livestock and Poultry Industry. 2019,30(9):33

2. Falowo A B, Akimoladun O F. Veterinary Drug Residues in Meat and meat products: occurrence, detection and implications[M]//Veterinary Medicine and Pharmaceuticals. IntechOpen, 2019: 53.

3. Clement M, Olabisi M, David E, et al. Veterinary pharmaceuticals and antimicrobial resistance in developing countries[M]//Veterinary Medicine and Pharmaceuticals. IntechOpen, 2019.

4. Charuaud L, Jardé E, Jaffrézic A, et al. Veterinary pharmaceutical residues from natural water to tap water: Sales, occurrence and fate[J]. Journal of hazardous materials, 2019, 361: 169-186.

5. Bottoni P, Caroli S. Presence of residues and metabolites of pharmaceuticals in environmental compartments, food commodities and workplaces: A review spanning the three-year period 20142016[J]. Microchemical Journal, 2018, 136: 2-24.

6. Teshome D. Review on rational use of veterinary antimicrobials and anthelmintics[J]. Austin Journal of Veterinary Science and Animal Husbandry, 2018, 5: 1044.

7. Gupta P. Concepts and applications in veterinary toxicology[J]. Cham (Switzerland): Springer International Publishing, 2019: 242-244.

8. Lee H C, Chen C M, Wei J T, et al. Analysis of veterinary drug residue monitoring results for commercial livestock products in Taiwan between 
2011 and 2015[J]. journal of food and drug analysis, 2018, 26(2): 565-571.

9. Zhang $\mathrm{S}$, Abbas $\mathrm{M}$, Rehman $\mathrm{M} \mathrm{U}$, et al. Dissemination of antimicrobial resistance genes (ARGs) via integrons in Escherichia coli: A risk to human health[J]. Environmental Pollution, 2020: 115260.

10. Lees P, Pelligand L, Giraud E, et al. A history of antimicrobial drugs in animals: Evolution and revolution[J]. Journal of Veterinary Pharmacology and Therapeutics, 2021, 44(2): 137-171.

11. Fatoki O S, Opeolu B O, Genthe B, et al. Multiresidue method for the determination of selected veterinary pharmaceutical residues in surface water around Livestock Agricultural farms[J]. Heliyon, 2018, 4(12): e01066.

12. Zeng Z, Yang F, Wang L. Veterinary Drug Residues in China[J]. Food Safety in China: Science, Technology, Management and Regulation, 2017: 219-235.

13. Zeinali T. Antibiotic Residues in foods; a public Health Hazard[J]. Journal of Health Sciences and Technology, 2017, 1(4): 205-206.

14. Jayalakshmi K, Paramasivam M, Sasikala M, et al. Review on antibiotic residues in animal products and its impact on environments and human health[J]. J Entomol Zool Stud, 2017, 5(3): 144651.

15. Xiaojie Z. Hazard and detection of veterinary drug residues in animal products[J]. The Chinese Livestock and Poultry Breeding ,2020,16(09):55.

16. Zhenwu Z. Hazards and detection methods of common veterinary drug residues in animal products[J]. Chinese Journal of Animal Husbandry and Veterinary Medicine, 2020(07):186.

17. Caisike A, Gaowa S. Harm and Causes of Veterinary Drug Residues in Animal Food [J].Graziery Veterinary Sciences(Electronic Version), 2020(14):156-157.

18. Rahman M M, Abd El-Aty A M, Kim S W, et al. Quick, easy, cheap, effective, rugged, and safe sample preparation approach for pesticide residue analysis using traditional detectors in chromatography: A review[J]. Journal of separation science, 2017, 40(1): 203-212.

19. Li Y. Analysis of the effect of veterinary drug residues on the animal-origin food safety based on statistical analysis [J]. China Food Safety Magazine, 2019(27):167.

20. Xue G. Effects of Veterinary Drug Residues on the Safety of Animal-origin Food [J]. Graziery Veterinary Sciences (Electronic Version), 2020(21):151-152.

21. Oliveira N A, Gonçalves B L, Lee S H I, et al. Use of antibiotics in animal production and its impact on human health[J]. Journal of Food
Chemistry and Nanotechnology, 2020, 6(01): 4047.

22. Ortelli D, Spörri A S, Edder P. Veterinary drug residue in food of animal origin in Switzerland: a health concern?[J]. CHIMIA International Journal for Chemistry, 2018, 72(10): 713-717.

23. Bacanlı M, Başaran N. Importance of antibiotic residues in animal food[J]. Food and Chemical Toxicology, 2019, 125: 462-466.

24. Amira M. Detection of Beta-lactam and Tetracycline Residues in Raw Dairy Milk in Biskra Region[J].University Mohamed Khider of Biskra.2020.1-40

25. Doña I, Torres $M J$, Montañez M I, et al. In vitro diagnostic testing for antibiotic allergy[J]. Allergy, asthma \& immunology research, 2017, 9(4): 288.

26. Roy D C, Kafle A. Antibiotic residue in animal foods: An alarm[J]. 2020,9(9):232-235

27. Topi D, Spahiu J. Presence of veterinary antibiotics in livestock manure in two Southeastern Europe countries, Albania and Kosovo[J]. Environmental Science and Pollution Research, 2020, 27(35): 44552-44560.

28. Jing G. Study on the detection technology of veterinary drug residues in food [J]. China Food Safety Magazine , 2020(09):162.

29. Xiaojuan S. Study on the determination of veterinary drug residues in food by mass spectrometry [J]. China Food Safety Magazine, 2018(06):77.

30. Yawen G,Xiaona B, Chujun L, Yajuan W, Bo W,Kaizhou X. Development in the determination of fluoroquinolones residues in food of animal origin by chromatography-mass spectrometry [J]. Chinese Journal of Veterinary Drug, 2019, 53(01):64-76.

31. Yu X, Lina G. Application of rapid detection technology in the detection of veterinary drug residues in egg products [J]. China Food Safety Magazine, 2020(09): 172.

32. Qihang C, Huiling W, Huitian G, Huiwen X. Research progress on detection technology of veterinary drug residues in food of animal origin [A]. Veterinary Food Hygiene Branch of Chinese Association of Animal Husbandry and Veterinary Medicine. Chinese Society of Animal Husbandry and Veterinary Medicine Veterinary Food Hygiene Branch 15th Academic Exchange Proceedings [C]. Chinese Association of Animal Husbandry and Veterinary Medicine Veterinary Food Hygiene Branch: Chinese Association of Animal Husbandry and Veterinary Medicine,2019:5.

33. Fang Y, Wendian M, Shengjian W, Fan Y. Application of liquid chromatography-mass spectrometry in the determination of veterinary drug residues [J]. Modern Food, 2020(09):185$186+190$. 
34. Lee H J, Cho S H, Shin D, et al. Prevalence of antibiotic residues and antibiotic resistance in isolates of chicken meat in Korea[J]. Korean journal for food science of animal resources, 2018, 38(5): 1055.

35. Runbo L, Yangdong Z, Nan Z, Jiaqi W. Research progress of high-throughput detection methods for veterinary drug residues in milk and dairy products $[\mathrm{J}]$, China Animal Husbandry and Veterinary Medicine,2020,47(10):3410-3416.

36. González-Gaya B, Cherta L, Nozal L, et al. An optimized sample treatment method for the determination of antibiotics in seawater, marine sediments and biological samples using LC$\mathrm{TOF} / \mathrm{MS}[\mathrm{J}]$. Science of the total environment, 2018, 643: 994-1004.

37. Zhao C, Junmin C, Yongsheng P. Current status and development direction of veterinary drug residue detection technology [J].Modern Food, 2017(14):12-14.

38. Silva J M, Azcárate F J, Knobel G, et al. Multiple response optimization of a QuEChERS extraction and HPLC analysis of diclazuril, nicarbazin and lasalocid in chicken liver[J]. Food chemistry, 2020, 311: 126014.

39. YUVALI D, Narin İ. Simultaneous determination of sulfachloropyridazine and trimethoprim in veterinary formulations by $\mathrm{hplc}[\mathrm{J}]$. Cumhuriyet Science Journal, 2020, 41(1): 239-244.

40. Juan C, Sanni L. Research on the determination method of pesticide residues in food $[\mathrm{J}]$. China Food, 2019(07):142.

41. Changwei W, Pipi Z. Analysis on the detection of pesticide and veterinary drug residues in food [J]. Animal Industry and Environment,2020(02):41.

42. Ji W, Jing Z, Yali X. Exploration of detection technology of pesticide and veterinary drug residues to build a strong defense line of food safety [J]. China Food, 2020(Z2):137.

43. Kabrite S, Bou-Mitri C, Fares J E H, et al. Identification and dietary exposure assessment of tetracycline and penicillin residues in fluid milk, yogurt, and labneh: A cross-sectional study in Lebanon[J]. Veterinary world, 2019, 12(4): 527.

44. Baghani A, Mesdaghinia A, Rafieiyan M, et al. Tetracycline and ciprofloxacin multiresidues in beef and chicken meat samples using indirect competitive ELISA[J]. Journal of Immunoassay and Immunochemistry, 2019, 40(3): 328-342.

45. Girmatsion M, Mahmud A, Abraha B, et al. Rapid detection of antibiotic residues in animal products using surface-enhanced Raman Spectroscopy: A review[J]. Food Control, 2021: 108019.

46. Tao W, Changwei W. Application of modern biological technology in the detection of pesticide and veterinary drug residues[J]. Animal Industry and Environment,2020(03):26.
47. Gaudin V, Hedou C, Soumet C, et al. Multiplex immunoassay based on biochip technology for the screening of antibiotic residues in milk: validation according to the European guideline $[\mathrm{J}]$. Food Additives \& Contaminants: Part A, 2018, 35(12): 2348-2365.

48. Aksoy A. Simultaneous screening of antibiotic residues in honey by biochip multi-array technology $[\mathrm{J}]$. Medycyna Weterynaryjna, 2019, 75(9): 6240-2019.

49. Shenling W, Guangyan P, Chuangang W, Kaixuan D, Zhenjia Z. Application of molecular imprinting technology in the detection of veterinary drug residues $[\mathrm{J}]$. Food Science and Technology, 2019,44(09):348-351.

50. Wenyu S, Tongcun Z. Research on the Purification of Arthromycin by Molecularimprinted Solid Phase Extraction [J]. Journal of Tianjin University of Science \& Technology, 2017,32(01):31-35.

51. Fang L, Huailin $\mathrm{K}$, Ruihua $\mathrm{Z}$, Hongyan $\mathrm{M}$. Research progress of biosensors for detection of pesticide residues in food [J]. Science and Technology of Food Industry,2017,38(04):396400.

52. Yu L. Application and development of biosensors in food safety detection $[\mathrm{J}]$. Modern Food, , 2019(06):26-28. 